\title{
EFFECTS OF NANO-POTASSIUM FERTILIZER ON AMINO ACID COMPONENTS AND GLN FAMILY GENE EXPRESSION IN CHINESE CABBAGE
}

\author{
JIN, Z. J. - LIU, Y. - XU, W. H. ${ }^{*}-$ YANG, C. - CHEN, S. L. - LI, Y. H. \\ College of Resources and Environmental Sciences, Southwest University, Chongqing 400715, \\ P. R. China \\ (phone: +86-23-68251249; fax: +86-23-68251912) \\ *Corresponding author \\ e-mail:xuwei_hong@163.com \\ (Received $15^{\text {th }}$ Feb 2020; accepted $7^{\text {th }}$ Jul 2020)
}

\begin{abstract}
This study was conducted to investigate the effects of nano potassium silicate on amino acid components and family gene expression of nitrogen metabolism-related glutamine synthetase (GS) in Chinese cabbage (Brassica rapa L. ssp. pekinensis). With ordinary potassium silicate (OKSi) and nano potassium silicate $(\mathrm{NKSi})$ as different potassium fertilizers, the experiment set four potassium levels $(\mathrm{K} 2 \mathrm{O})$ of $0(\mathrm{CK}), 150,300$ and $450 \mathrm{~kg} \cdot \mathrm{hm}-2$, respectively. Field experiments were adopted to investigate the effects of different forms and levels of potassium on the amino acid content of Chinese cabbage and GLN family gene expression in roots, stems and leaves. The total amount of essential amino acids was the highest in NKSi-300 treatment, which was $41.1 \%$ higher than that in the control (CK). In the stem of Chinese cabbage, the relative expression of GLN1.2, GLN1.3, GLN1.4 and GLN2 was up by 7.85, 3.46, 45.6 and 50.7 times compared with CK under NKSi-300 treatment. In Chinese cabbage leaves, GLN1.1, GLN1.2 and GLN1.3 had the highest relative expressions under NKSi-150 treatment, which were up by 1.984, 1.235 and 1.617 times, respectively compared to CK. GLN family gene expression was significantly or extremely significantly correlated with histidine, glycine, methionine, tyrosine and cysteine contents in the stem of Chinese cabbage.
\end{abstract}

Keywords: nano potassium silicate, Chinese cabbage, amino acid, GLN family gene, correlation analysis

\section{Introduction}

It has become an important goal to improve vegetable quality in today's vegetable production. Amino acids, sugars, organic acids and volatile metabolites have always acted as important indicators for evaluating nutritional and flavor quality of vegetables. To study the key enzymes and key genes involved in the synthesis of these compounds carries important significance for vegetable quality control. As one of the essential nutrients for plant growth and development, potassium participates in multiple metabolic processes of plants, which is crucial for the development of quality vegetable. However, China lacks potash fertilizer resources, potassium-ion soil deficiency is prevalent in China and Asia, and urgent solution is needed. Nanotechnology involves the understanding and control of substances at the nanoscale, i.e. new applications having unique physical, chemical and biological properties (nanomaterials, NMs) with dimensions in the range of 1-100 nm (Huang et al., 2014). Nano-fertilizers provide certain effects in improving fertilizer utilization rate and agricultural product quality. Nano-selenium fertilizer can increase the content of selenomethionine, selenium methylation cysteine, etc. in wheat (Li et al., 2017). The experiment results on combined application of low potassium and nano magnesium hydroxide showed significantly increased contents of carotene, total phenols and flavonoids in Chinese cabbage (Brassica rapa L. ssp. pekinensis), with potassium utilization efficiency significantly improved (Yuan et al., 2017). Foliar spray 
tests on nano chelate nitrogen fertilizer and urea showed that the former can better improve the quality of pomegranate fruit (Davarpanah et al., 2017).

Since the 1930s, breeders have been emphasizing yield increase, basically ignoring vegetable quality in aspects like flavors and nutritional properties of interest to consumers (Gascuel et al., 2017). For the reason: first, it is quite difficult to obtain complex polygenic traits such as flavor; second, we lack understanding of molecular genetic basis of vegetable quality (Lim et al., 2014). These result in decline in vegetable quality and loss of flavor, indirectly producing a negative impact on vegetable consumption. Despite the continuous development of biotechnology in recent decades, breeding programs often fail in the face of complex quality traits (Mattoo et al., 2014). Advances in biotechnology and omics technology for variant traits may help us decode the underlying genetic basis of complex traits, so that it is possible to implant optimal allele to vegetables by hybridization, genetic engineering, transgenic techniques or new plant breeding techniques (NPBT) to improve vegetable quality (Gascuel et al., 2017). To this end, this study adopted field experiments to investigate the effect of nano potassium silicate on amino acid composition of Chinese cabbage, and explore the effect of nano potassium silicate on the family gene expression of amino acid metabolism and nitrogen metabolism-related glutamine synthetase in Chinese cabbage. The study aims to clarify the physiological and molecular biological mechanism in amino acid metabolism regulation of vegetables by nano-potassium fertilizer, thus providing a theoretical basis for efficient utilization of potassium fertilizer resources and high-quality vegetable production.

\section{Materials and methods}

\section{Plant material, soil and treatments}

The Chinese cabbage (Brassica rapa L. ssp. pekinensis) variety for the field experiment was "Liangqing", and the test fertilizer was the same as that in the pot experiment. The physical and chemical properties of the tested soil are shown in Table 1.

Table 1. Basic soil physical and chemical properties in the field experiment

\begin{tabular}{|c|c|c|c|c|c|}
\hline $\begin{array}{c}\mathrm{pH} \\
\text { Water/Soil=1/1 }\end{array}$ & $\begin{array}{c}\text { Organic } \\
\text { matter } / \mathbf{g} \cdot \mathbf{k g}^{-1}\end{array}$ & $\begin{array}{c}\text { Total } \\
\text { nitrogen } \\
/ \mathbf{g} \cdot \mathbf{k g}^{-1}\end{array}$ & $\begin{array}{c}\text { Available } \\
\text { nitrogen } / \mathrm{mg}_{1} \cdot \mathrm{kg}^{-}\end{array}$ & $\begin{array}{c}\text { Available } \\
\text { phosphorus } \\
/ \mathbf{m g} \cdot \mathbf{k g}^{-1}\end{array}$ & 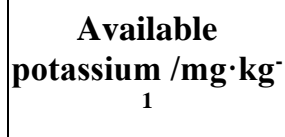 \\
\hline 5.80 & 25.8 & 1.70 & 140 & 127 & 77.5 \\
\hline
\end{tabular}

The field experiment was conducted from February 18, 2017 to May 19, 2017 at Sanyuan Village, Badang Town, Bishan District, Chongqing, China. There were three potassium levels (K2O), 150, 300 and $450 \mathrm{~kg} \cdot \mathrm{hm}^{-2}$, respectively, as well as potassiumfree blank control (CK) (Table 2). Each treatment was repeated 3 times, and application of nitrogen $(\mathrm{N})$ and phosphorus (P2O5) was consistent in all treatments. According to the customary application rate of farmers, $750 \mathrm{~kg} \cdot \mathrm{hm}^{-2}$ nitrogen-phosphorus compound fertilizer (15-15-0) was converted into urea and superphosphate and applied to the soil by means of furrow application. Only nitrogen fertilizer was applied in late topdressing, and the dosage was $300 \mathrm{~kg} \cdot \mathrm{hm}^{-2}$ urea. The test plot area was $2 \mathrm{~m} \times 5 \mathrm{~m}=10 \mathrm{~m}^{2}$, a total of 21 plots. 60 strains of Chinese cabbage seedlings with uniform growth conditions were 
transplanted in each plot, arranged in random blocks, with a spacing of $0.5 \mathrm{~m}$ between the plots for easy management. Also, guard rows were set around. Field management was carried out in accordance with conventional management. After the Chinese cabbage matured, 5 representative plants were selected and harvested from each plot. Different organ samples of Chinese cabbage were collected by portable liquid nitrogen tanks and stored in a $-80{ }^{\circ} \mathrm{C}$ ultra-low temperature refrigerator for subsequent gene expression determination. Part of the harvested plants were used for determination of vegetable quality indicators, while part was killed at $105^{\circ} \mathrm{C}$, dried, crushed and stored at $65-80{ }^{\circ} \mathrm{C}$ for analysis and detection of plant nutrients.

Table 2. Field experiment design and fertilization amount

\begin{tabular}{|c|c|}
\hline Treatment & $\mathrm{N}-\mathrm{P}_{2} \mathrm{O}_{5}-\mathrm{K}_{2} \mathrm{O} / \mathrm{kg} \cdot \mathrm{hm}^{-2}$ \\
\hline $\mathrm{CK}$ & $112.5-112.5-0$ \\
\hline OKSi-150 & $112.5-112.5-150$ \\
\hline OKSi-300 & $112.5-112.5-300$ \\
\hline OKSi-450 & $112.5-112.5-450$ \\
\hline NKSi-150 & $112.5-112.5-150$ \\
\hline NKSi-300 & $112.5-112.5-300$ \\
\hline NKSi-450 & $112.5-112.5-450$ \\
\hline
\end{tabular}

\section{Amino acid component determination}

Measurement was carried out using L-8800 automatic amino acid analyzer (Hitachi, Japan).

\section{RNA extraction}

RNA was extracted from the roots, stems and leaves of Chinese cabbage using EZ-10 DNAaway RNA Mini-Preps Kit (Sangon Biotech Co., Ltd, China). Please refer to the operating instruction on the kit for the specific procedures. The sample RNA was stored in $-80{ }^{\circ} \mathrm{C}$ refrigerator for later use.

\section{RNA concentration and quality detection}

Using RNase-Free Water as a reference, the concentration and quality of extracted RNA were detected using a NanoDrop 2000C spectrophotometer (500 ng/band) (Thermo Scientific, Wilmington, DE, USA), and RNA degradation degree and quality were measured by $1 \%$ agarose gel electrophoresis. The results are shown in Table 3 and Figure 1.

The detection results of NanoDrop 2000C spectrophotometer show that RNA concentration is greater than $125 \mathrm{ng} \cdot \mu \mathrm{L}^{-1}$, OD260/280 is between $1.8-2.21$, and OD260/OD230 is greater than 2.0, indicating that there is no protein or phenolic substances, carbohydrate, salt pollutions and quality and concentration are qualified.

The results of RNA agarose gel electrophoresis (Figure 1) show that $28 \mathrm{~S}$ band is brighter than $18 \mathrm{~S}$ band, $5 \mathrm{~S}$ band is the darkest or invisible, the characteristic bands are clear, the degradation is unobvious. Quality and concentration are assessed as qualified by luminance method ( $\mathrm{Li}$ et al., 2018) and usage for follow-up experiments like reverse transcription is possible. 
Table 3. Concentration and quality test results of RNA extracted from Chinese cabbage in the field experiment

\begin{tabular}{c|c|c|c|c}
\hline No. & Treatments & $\begin{array}{c}\text { RNA concentrations } \\
/ \mathbf{n g} \cdot \boldsymbol{\mu L}^{\mathbf{1}}\end{array}$ & OD $_{\mathbf{2 6 0}} / \mathbf{O D}_{\mathbf{2 8 0}}$ & $\mathbf{O D}_{\mathbf{2 6 0}} / \mathbf{O D}_{\mathbf{2 3 0}}$ \\
\hline 1 & CK-BrR & 672.4 & 2.17 & 2.32 \\
2 & OKSi-150-BrR & 815.8 & 2.18 & 2.38 \\
3 & OKSi-300-BrR & 565.0 & 2.17 & 2.29 \\
4 & OKSi-450-BrR & 1172.4 & 2.21 & 2.36 \\
5 & NKSi-150-BrR & 492.8 & 2.14 & 2.19 \\
6 & NKSi-300-BrR & 1056.3 & 2.19 & 2.33 \\
7 & NKSi-450-BrR & 560.6 & 2.15 & 2.24 \\
8 & CK-BrS & 224.8 & 2.16 & 2.17 \\
9 & OKSi-150-BrS & 232.4 & 2.17 & 2.25 \\
10 & OKSi-300-BrS & 212.7 & 2.17 & 2.23 \\
11 & OKSi-450-BrS & 213.9 & 2.18 & 2.23 \\
12 & NKSi-150-BrS & 126.3 & 2.17 & 2.14 \\
13 & NKSi-300-BrS & 140.5 & 2.18 & 2.14 \\
14 & NKSi-450-BrS & 135.9 & 2.19 & 2.09 \\
15 & CK-BrL & 1348.1 & 2.19 & 2.34 \\
16 & OKSi-150-BrL & 1629.1 & 2.2 & 2.35 \\
17 & OKSi-300-BrL & 1210.5 & 2.2 & 2.33 \\
18 & OKSi-450-BrL & 1071.3 & 2.21 & 2.37 \\
19 & NKSi-150-BrL & 1076.5 & 2.16 & 2.27 \\
20 & NKSi-300-BrL & 1894 & 2.16 & 2.27 \\
21 & NKSi-450-BrL & 847.2 & 2.16 & 2.22 \\
\hline
\end{tabular}

Note: $\mathrm{BrR} / \mathrm{S} / \mathrm{L}$ indicates the root/stem/leaf of Chinese cabbage, the same below

\section{RNA purification and reverse transcription}

gDNA removal and reverse transcription were performed using TaKaRa PrimeScript ${ }^{\circledR}$ Reagent Kit. For specific procedures, refer to the kit's operating instructions. The reverse transcription product cDNA was stored in a $-20^{\circ} \mathrm{C}$ refrigerator.

\section{Detection of reverse transcription product cDNA}

With cDNA as a template, F26Sq+R26Sq was used as a primer (primer sequence was 5'-GTTACCACAGGGATAACTGGCTTG-3' + 5'-CTAACCTGTCTCACGACGG TCTAA-3'), and 26SrRNA gene was amplified by $25 \mu \mathrm{L}$ system and detected by agarose gel electrophoresis. The reaction system is as follows (Table 4).

The PCR reaction procedure was as follows: predenaturation at $94{ }^{\circ} \mathrm{C}$ for $2 \mathrm{~min}$; denaturation at $94{ }^{\circ} \mathrm{C}$ for $1 \mathrm{~min}$, annealing at $60{ }^{\circ} \mathrm{C}$ for $30 \mathrm{~s}$, extension at $72{ }^{\circ} \mathrm{C}$ for $30 \mathrm{~s}$, repetition by 18 cycles and 35 cycles; extension at $72{ }^{\circ} \mathrm{C}$ for $10 \mathrm{~min}$ and at $4{ }^{\circ} \mathrm{C}$ for $2 \mathrm{~min}$.

The results of cDNA electrophoresis (Figure 2) show that a clear band of about $173 \mathrm{bp}$ is obtained by agarose gel electrophoresis except for No.6 (Figure 2A), indicating successful reverse transcription. However, RNA reverse transcription efficiency differs due to different RNA concentrations and purities, so $173 \mathrm{bp}$ bands show slightly different brightness, and No.6 only has weak brightness. As can be seen from Figure 2B, No. 6 has been reverse transcribed successfully, though the reverse transcription efficiency is low. 


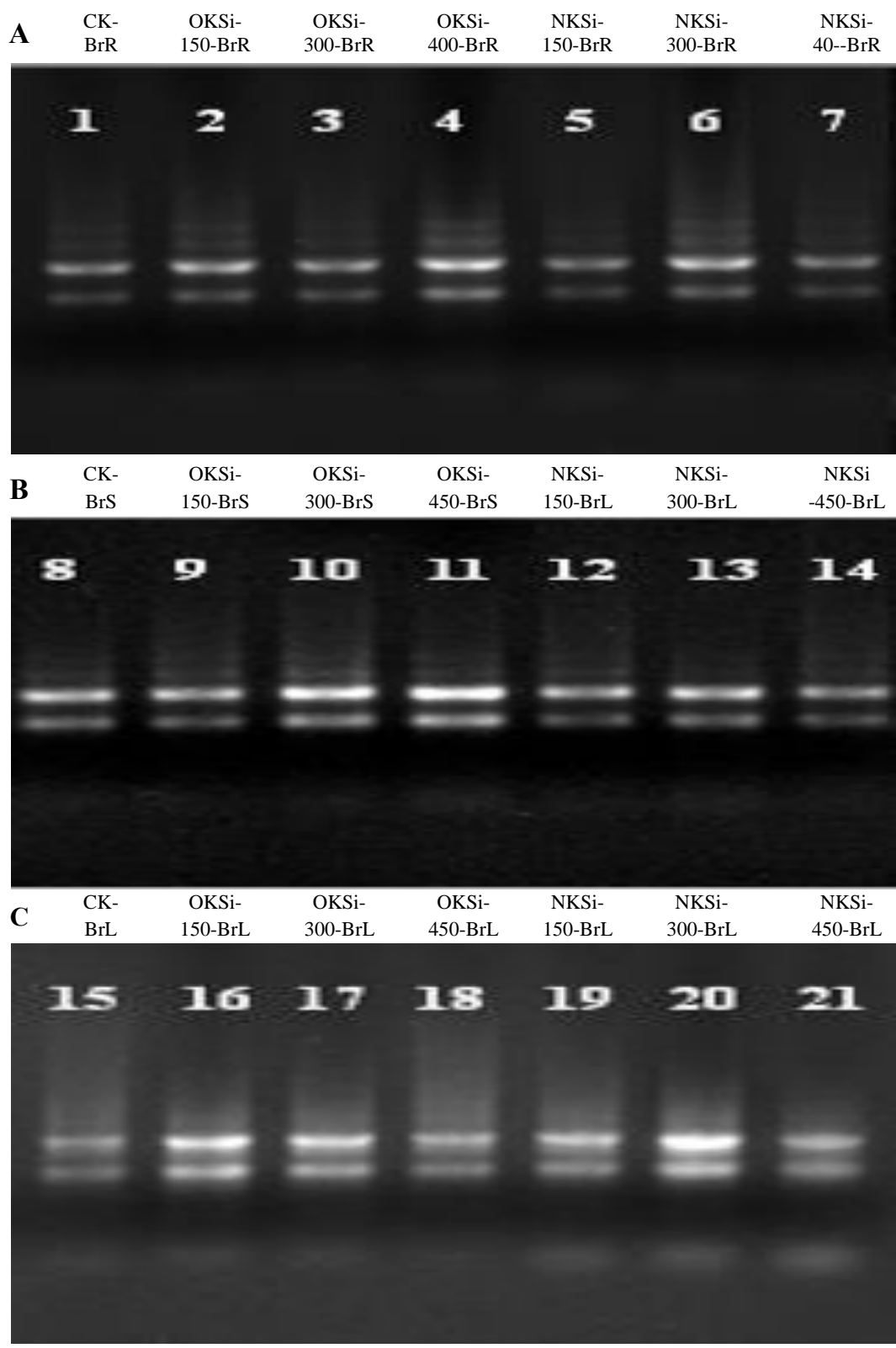

Figure 1. Agarose gel electrophoresis detection results of RNA extracted from Chinese cabbage. Note: No.1-21 has the same meaning as Table 3, M-Trans $2 \mathrm{~K}$ Plus Marker

Table 4. Amplification system of reverse transcription product PCR

\begin{tabular}{c|c}
\hline Reagents & Volume $/ \boldsymbol{\mu L}$ \\
\hline $\mathrm{ddH} \mathrm{H}_{2} \mathrm{O}$ & 20.15 \\
$\mathrm{cDNA}$ & 0.50 \\
$\mathrm{dNTPS} / 10 \mathrm{mmoL} \cdot \mathrm{L}^{-1}$ & 0.50 \\
$\mathrm{~F} 26 \mathrm{Sq} / 10 \mu \mathrm{moL} \cdot \mathrm{L}^{-1}$ & 0.50 \\
$\mathrm{R} 26 \mathrm{Sq} / 10 \mu \mathrm{moL} \cdot \mathrm{L}^{-1}$ & 0.50 \\
$10 \times$ Easy Taq Buffer $\left(\mathrm{Mg}^{2+}\right)$ & 2.50 \\
Easy-Taq DNA PoLymerase $/ 5 \mathrm{U} \cdot \mu \mathrm{L}^{-1}$ & 0.35 \\
\hline Total volume & 25.0 \\
\hline
\end{tabular}

Note: F-Forward primer, R-Reverse primer 


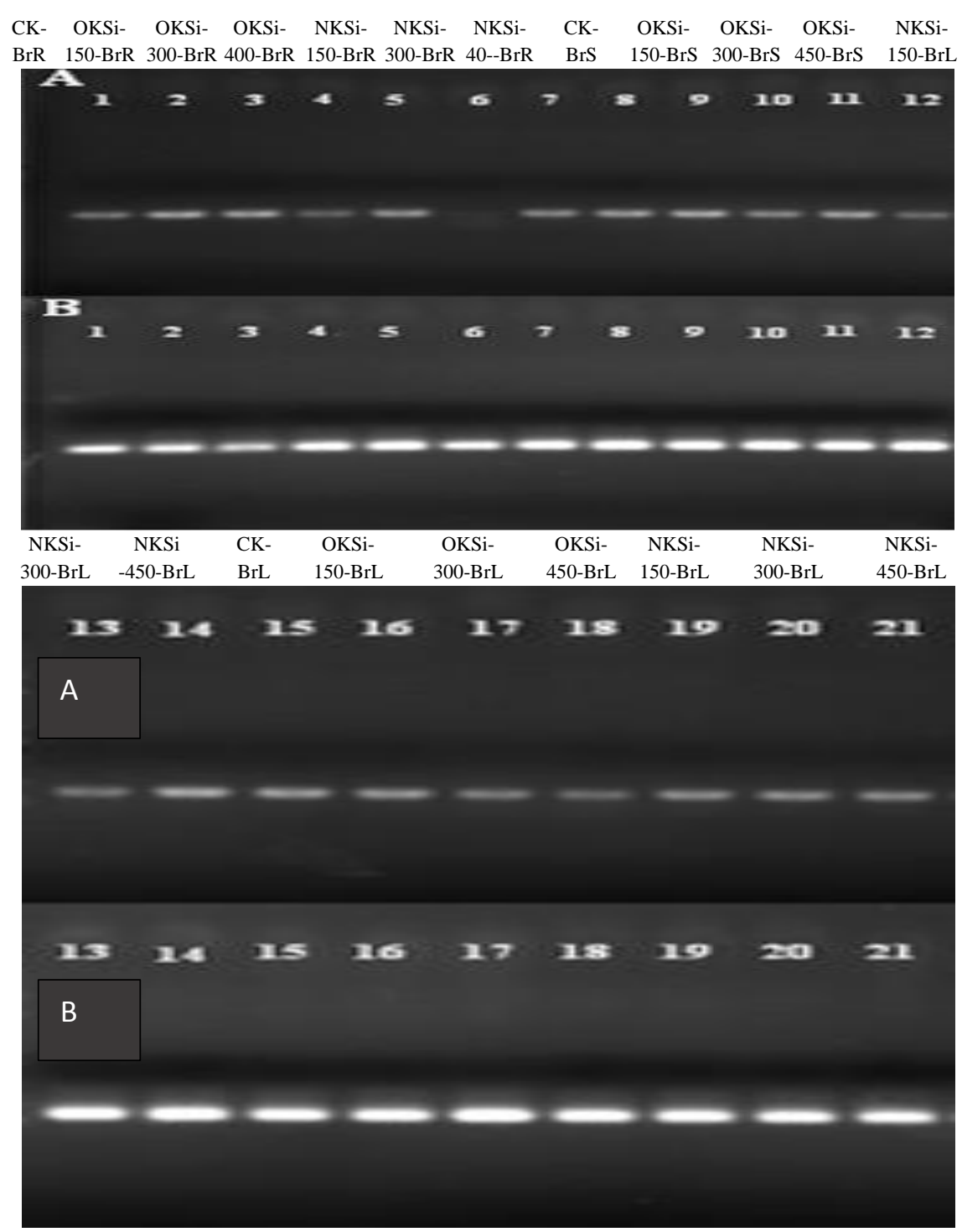

Figure 2. Agarose gel electrophoresis results of reverse transcription product cDNA. Note: 18A-PCR amplified cyclic electrophoresis results; 35 B-PCR amplified cyclic electrophoresis results, number 1-21 has the same meaning as those in Table 3, M-Trans 2K Plus Marker

\section{Electronic cloning of GLN family gene}

The locus number of the Arabidopsis thaliana L. GLN family gene was accessed from Arabidopsis thaliana L. database. BLAST was performed on NCBI website, Organism was defined as Arabidopsis thaliana L. and Brassica rapa L. ssp. pekinensis, respectively. The database was defined as Reference RNA sequence (refseq_rna), Reference genomic sequences (refseq_ genomic) and Transcriptome Shotgun Assembly (TSA). The reference RNA sequences, reference chromosomal sequences and TSA sequences of Arabidopsis thaliana L. and Brassica L. ssp. pekinensis GLN family genes were obtained from BLAST results; sequences were created using Vector NTI Advance 11.5. Then, using the Arabidopsis thaliana L. GLN family gene sequence as a basic sequence, the above sequence tags were added one by one for alignment until multiple alignments were completed. 


\section{GLN family gene primer design}

Based on the electronic cloning results of GLN family genes, corresponding qRT-PCR primers were designed based on sequence difference sites between the species (Table 5).

Table 5. $q R T-P C R$ primers of GLN family gene of Chinese cabbage

\begin{tabular}{c|c|c}
\hline Primers & Primer Sequence $\left(\mathbf{5}^{\prime} \rightarrow \mathbf{3}^{\prime}\right.$ ) & Predicted annealing temperature \\
\hline FBrGLN1.1 & CTGGCATCAACATTAGTGGCATC & 60.0 \\
RBrGLN1.1 & TGTGTCTCAGTCCCAATTTATCG & 60.0 \\
FBrGLN1.2 & GTATGCTGGAATTAACATCAGTGG & 62.0 \\
RBrGLN1.2 & CTTGATTATCTCGTATCCTCCTTC & 62.0 \\
FBrGLN1.3 & TCCGACCAACAAGAGGCACAA & 62.0 \\
RBrGLN1.3 & CGAAGCTGACATTTACACCAGAGA & 62.0 \\
FBrGLN1.4 & GTCTTTACGCCGGAATCAATGT & 62.0 \\
RBrGLN1.4 & GTGTTCCTTGTGACGCAATCCA & 62.0 \\
FBrGLN2 & CAGGTGATCATGTTTGGTGTGC & 62.0 \\
FBrGLN2 & TGCTTTCCGGTCAACCTTCTC & 62.0 \\
\hline
\end{tabular}

Note: F-forward primer, R-reverse primer, Br-Chinese cabbage, GLN-glutamine synthetase

\section{Primer annealing temperature gradient test}

The mixed cDNA of Chinese cabbage in each treatment was used as a template, and six temperature gradients at $55^{\circ} \mathrm{C}, 57^{\circ} \mathrm{C}, 59{ }^{\circ} \mathrm{C}, 60{ }^{\circ} \mathrm{C}, 61^{\circ} \mathrm{C}$ and $62{ }^{\circ} \mathrm{C}$ were set for the combination of (forward primer and reverse primer) in Table 2 with $60{ }^{\circ} \mathrm{C}$ as the criterion. The $25 \mu \mathrm{L}$ standard semi-quantitative RT-PCR system was used to amplify the GLN family genes in each sample at different temperatures. The amplified products were subjected to $1 \%$ agarose gel electrophoresis to detect the optimum annealing temperature for each primer. The reaction system is as follows (Table 6):

Table 6. Primer annealing temperature gradient test on PCR amplification system

\begin{tabular}{c|c}
\hline Reagents & Volume $/ \boldsymbol{\mu L}$ \\
\hline $\mathrm{ddH} \mathrm{H}_{2} \mathrm{O}$ & 11.0 \\
$\mathrm{cDNA}$ & 0.5 \\
Forward primers $/ 10 \mu \mathrm{mol} \cdot \mathrm{L}^{-1}$ & 0.5 \\
Reverse primers $/ 10 \mu \mathrm{mol} \cdot \mathrm{L}^{-1}$ & 0.5 \\
FastStart Essential DNA Green Master & 12.5 \\
\hline Total volume & 25.0 \\
\hline
\end{tabular}

PCR reaction procedure was as follows: pre-denaturation at $94{ }^{\circ} \mathrm{C}$ for $2 \mathrm{~min}$; denaturation at $94{ }^{\circ} \mathrm{C}$ for $1 \mathrm{~min}$, annealing at $\mathrm{T0}{ }^{\circ} \mathrm{C}$ for $30 \mathrm{~s}$, extension at $72{ }^{\circ} \mathrm{C}$ for $30 \mathrm{~s}$, repetition for 40 cycles; extension at $72{ }^{\circ} \mathrm{C}$ for $10 \mathrm{~min}$ and at $4{ }^{\circ} \mathrm{C}$ for $2 \mathrm{~min}\left(\mathrm{~T} 0{ }^{\circ} \mathrm{C}\right.$ means predicted annealing temperature of different primers). GLN1.1-GLN1.4 electrophoresis bands of Chinese cabbage had gradually increased brightness with increasing temperature, reaching the top at $62{ }^{\circ} \mathrm{C}$, and GLN2 bands basically had no differences in brightness. Therefore, the annealing temperature of Chinese cabbage GLN family was set at $62{ }^{\circ} \mathrm{C}$ (Figure 3 ) and the annealing temperature of qRT-PCR could be uniformly set to $62{ }^{\circ} \mathrm{C}$. 


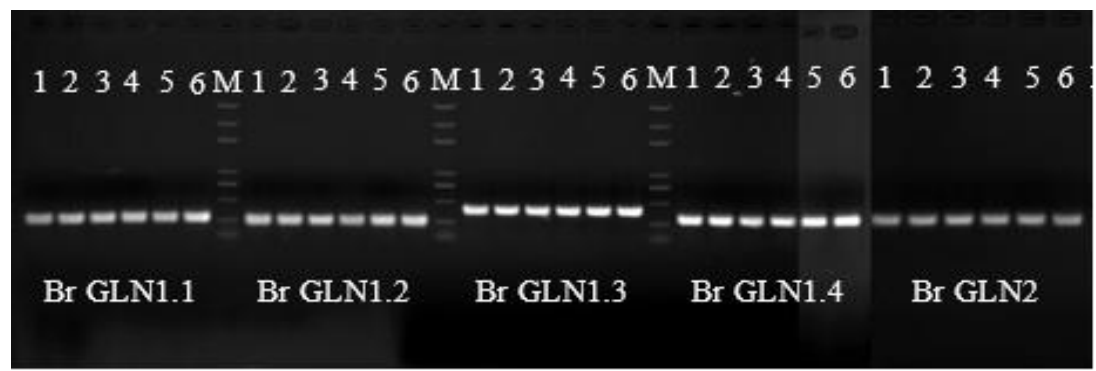

Figure 3. Agarose gel electrophoresis detection results of Chinese cabbage GLN family gene annealing temperature gradient test. Note: $1: 55^{\circ} \mathrm{C}, 2: 57^{\circ} \mathrm{C}, 3: 59^{\circ} \mathrm{C}, 4: 60^{\circ} \mathrm{C}, 5: 61^{\circ} \mathrm{C}, 6: 62^{\circ} \mathrm{C}, \mathrm{M}$ :

\section{qRT-PCR detection of GLN family gene}

The cDNA of reverse transcription products of Chinese cabbage in 7 different treatments was diluted 30-fold as a template, 26SrRNA was used as an internal reference gene, and (forward primer and reverse primer) in Table 2 was used as a combination to detect expression differences of Chinese cabbage GLN family genes in different organs under different treatments by qRT-PCR (CFX96TM Real-Time System, New England Biolabs, USA). Each treatment of each gene was repeated by 3 times. The reaction system is as follows (Table 7):

Table 7. $q R T-P C R$ reaction system

\begin{tabular}{c|c}
\hline Reagents & Volume $/ \mu \mathrm{L}$ \\
\hline ddH ${ }_{2} \mathrm{O}$ & 1.5 \\
cDNA & 2.5 \\
Forward primers $/ 10 \mu \mathrm{mol} \cdot \mathrm{L}^{-1}$ & 0.5 \\
Reverse primers $/ 10 \mu \mathrm{mol} \cdot \mathrm{L}^{-1}$ & 0.5 \\
FastStart Essential DNA Green Master & 5.0 \\
\hline Total volume & 10.0 \\
\hline
\end{tabular}

qRT-PCR reaction procedure was as follows: pre-denaturation at $95{ }^{\circ} \mathrm{C}$ for $10 \mathrm{~min}$; denaturation at $95{ }^{\circ} \mathrm{C}$ for $10 \mathrm{~s}$, annealing at $\mathrm{T} 1{ }^{\circ} \mathrm{C}$ for $30 \mathrm{~s}$, repetition by 45 cycles; addition of melting curve ranging from $65^{\circ} \mathrm{C}$ to $95^{\circ} \mathrm{C}\left(\mathrm{T} 1{ }^{\circ} \mathrm{C}\right.$ was the optimum annealing temperature for different primers).

\section{Statistical analysis}

Three-way analysis of univariate ANOVA and correlation analysis were performed using SPSS version 21.0package (SPSS, 2009).

\section{Results and analysis}

\section{Amino acid component}

As can be seen from Table 8, essential amino acids (BI), total non-essential amino acids (BD) and total amino acids (BT) of Chinese cabbage increase with the increasing application of ordinary potassium silicate $(\mathrm{OKSi})$ and nano potassium silicate $(\mathrm{NKSi})$. The total amount of essential amino acids is the highest in NKSi-300 treatment, which is 
$41.1 \%$ higher than that of control (CK), followed by OKSi-450 which is by $31.0 \%$ higher than that of the control. Both OKSi and NKSi treatments improve lysine (Lys) content, but NKSi has greater improvement than OKSi under the same application rate. Under 150,300 and $450 \mathrm{~kg} \cdot \mathrm{hm}^{-2}$ application rate, OKSi-Lys is increased by $33.4 \%, 45.5 \%$ and $64.3 \%$, respectively compared with control, NKSi-Lys is increased by $104 \%, 170 \%$ and $132 \%$, respectively compared with control, and NKSi-Lys is $53.2 \%, 85.3 \%$ and $41.5 \%$ higher than OKSi-Lys, respectively. OKSi treatment increases methionine (Met) content and there is greater increase with the increasing application rate of $\mathrm{OKSi}$, while $\mathrm{NKSi}$ demonstrate an effect opposite to that of OKSi. Met content is decreased with the increasing application rate. At application rates of 150,300 and $450 \mathrm{~kg} \cdot \mathrm{hm}^{-2}$, OKSi-Met is increased by $33.1 \%, 35.2 \%$ and $101 \%$ respectively compared with the control, while NKSi-Met is decreased by $53.8 \%, 57.9 \%$ and $65.5 \%$, respectively compared with the control. The ratio of essential amino acid to total amino acid (BI/BT) shows an upward trend, reaching the highest in NKSi-300 treatment, which is $22.3 \%$ higher than CK. Nonessential amino acid ratio (BD/BT) shows an opposite effect, with the lowest in NKSi300 treatment, which is $11.3 \%$ lower than CK. The ratios of total essential amino acids and non-essential amino acids (BI/BD) in adult are higher than that of $\mathrm{CK}$ under potassium silicate treatment, with $\mathrm{NKSi}-\mathrm{BI} / \mathrm{BD}$ higher than $\mathrm{OKSi}-\mathrm{BI} / \mathrm{BD}$ at the same application rate. NKSi-300 has the highest ratio, which is $37.7 \%$ higher than CK.

The above analysis shows that application of both OKSi and NKSi can increase amino acid content of Chinese cabbage, with superior effect in NKSi than OKSi.

\section{Expression characteristics of GLN family gene}

As can be seen from Figure 4, GLN family gene in Chinese cabbage root (BrR) shows the following tendency under potassium silicate treatment: under ordinary potassium silicate (OKSi) treatment, expression of GLN1.1, GLN1.2, GLN1.3 and GLN2 is first down-regulated and then up-regulated, with the highest expression under OKSi-450 treatment, which is up by $1.84,5.47,5.20$ and 5.57 times, respectively, compared with control (CK). Expression of GLN1.4 is gradually up-regulated with the increasing OKSi application rate, but the increase range is first increased and then increased, which is up by 4.37, 1.25 and 99.5 times respectively compared with CK under 150, 300 and $450 \mathrm{~kg} \cdot \mathrm{hm}^{-2}$ application rates. Under nano potassium silicate (NKSi) treatment, GLN1.1 and GLN1.2 expressions are only up-regulated under $300 \mathrm{~kg} \cdot \mathrm{hm}^{-2}$ treatment, which are up by 34.4 and 40.7 times, respectively compared with CK. GLN1.3 and GLN2 expressions are up-regulated at 300 and $450 \mathrm{~kg} \cdot \mathrm{hm}^{-2}$ treatments, up by $119,1.91$ and 67.2 , 0.108 times, respectively compared with CK. GLN1.4 is up-regulated under 150, 300 and $450 \mathrm{~kg} \cdot \mathrm{hm}^{-2}$ treatments, up by $9.57,1305$ and 6.48 times compared with CK, respectively. Compared with CK, GLN1.1, GLN1.2, GLN1.3, GLN1.4 and GLN2 genes have the highest relative expression under the treatment of $300 \mathrm{~kg} \cdot \mathrm{hm}^{-2} \mathrm{NKSi}$, showing the order of expression of GLN1.3 (158)> GLN1. 1 (154)>GLN1.4 (139)>GLN2 (125)>GLN1.2 (82.0). GLN1.1, GLN1.2, GLN1.3 and GLN2 expressions are down-regulated under $150 \mathrm{~kg} \cdot \mathrm{hm}^{-2}$ application rate, but OKSi has greater down-regulation than NKSi. On the other hand, GLN1.4 expression is up-regulated, but NKSi brings greater up-regulation effect than OKSi. Under $450 \mathrm{~kg} \cdot \mathrm{hm}^{-2}$ application rate, all of OKSi-GLN1.1, GLN1.2, GLN1.3, GLN1.4 and GLN2 show up-regulated expressions, NKSi-GLN1.1 and GLN1.2 show down-regulated expression, while GLN1.3, GLN1.4 and GLN2 show up-regulated expressions. The expression levels of each gene are higher in OKSi treatment than in NKSi treatment at $450 \mathrm{~kg} \cdot \mathrm{hm}^{-2}$ potassium feitilizer treatment. 
Table 8. The amino acid component and content in the aboveground parts of Chinese cabbage treated with potassium silicate

\begin{tabular}{|c|c|c|c|c|c|c|c|c|c|c|c|c|c|c|c|c|c|c|c|c|c|c|c|}
\hline \multirow{2}{*}{ Treatments } & Lys* & Met* & Ile* & Leu* & Phe* & $\mathrm{Val}^{*}$ & Thr* & $\mathrm{BI}$ & His & Arg & Glu & Ser & Gly & Ala & Tyr & Pro & Asp & Cys & $\mathrm{BD}$ & BT & BI/BT & $\mathrm{BD} / \mathrm{BT}$ & \multirow{2}{*}{$\mathrm{BI} / \mathrm{BD}$} \\
\hline & \multicolumn{20}{|c|}{$\mathrm{g} \cdot \mathrm{kg}^{-1} \mathrm{DW}$} & \multicolumn{2}{|c|}{$\% \mathrm{DW}$} & \\
\hline CK & 3.14 & 1.45 & 5.11 & 8.03 & 5.76 & 4.77 & 3.66 & 31.9 & 1.85 & 2.43 & 23.8 & 4.71 & 5.15 & 6.67 & 1.91 & 1.8 & 9.36 & 4 & 58.1 & 90.1 & 35.4 & 64.5 & 0.549 \\
\hline OKSi-150 & 4.19 & 1.93 & 5.86 & 9.01 & 6.02 & 6.23 & 4.41 & 37.7 & 2.02 & 2.64 & 25.7 & 5.40 & 5.71 & 7.36 & 2.37 & 6.20 & 10.4 & 0.640 & 68.5 & 106 & 35.6 & 64.6 & 0.550 \\
\hline NKSi-150 & 6.42 & 0.670 & 4.57 & 7.80 & 4.95 & 5.64 & 3.63 & 33.7 & 2.28 & 2.6 & 15.1 & 3.23 & 5.65 & 7.25 & 0.960 & 1.27 & 8.72 & 0.280 & 47.4 & 81.1 & 41.6 & 58.4 & 0.711 \\
\hline OKS & 4.57 & 1.96 & 5.60 & 9.18 & 5.50 & 5.99 & 4.13 & 36.9 & 1.98 & 2.41 & 23.1 & 4.77 & 5.39 & 6.55 & 2.17 & 5.71 & 10.2 & 0.590 & 62.8 & 100 & 36.9 & 62.8 & 0.588 \\
\hline NKSi-300 & 8.47 & 0.610 & 6.05 & 10.6 & 7.00 & 7.44 & 4.75 & 45.0 & 3.07 & .59 & 17.2 & 4.61 & 7.34 & 8.51 & 1.34 & 1.55 & 11.9 & 0.321 & 59.5 & 104 & 43.3 & 57.2 & 0.756 \\
\hline OKSi-450 & 5.16 & 2.92 & 5.99 & 9.98 & 6.65 & 6.48 & 4.59 & 41.8 & 2.44 & 2.75 & 24.5 & 4.84 & 5.95 & 7.76 & 3.01 & 1.81 & 10.6 & 0.660 & 64.3 & 106 & 39.4 & 60.7 & 0.650 \\
\hline NKSi-450 & 7.30 & 0.500 & 5.43 & 8.91 & 5.71 & 6.63 & 5.21 & 39.7 & 2.64 & 3.49 & 20.8 & 4.75 & 6.82 & 8.90 & 0.920 & 1.35 & 12.00 & 0.360 & 62.1 & 102 & 38.9 & 60.9 & 0.640 \\
\hline
\end{tabular}

Note: "*" means essential amino acids for adults, “»" means conditionally essential amino acids; Lys-lysine, Met-methionine, Ile-isoleucine, Leu-leucine, Phephenylalanine, Val-valine, Thr-threonine, BI-total essential amino acids in adults; His-histidine, Arg-arginine, Glu-glutamic acid, Ser-serine, Gly-glycine, Ala-alanine, Tyr-tyrosine, Pro-proline, Asp-asparaginic acid, Cys-cysteine, BD-total non-essential amino acids in adults, BT-total amino acid; BI/BT-A ratio of total essential amino acids in adults to total amino acids, BD/BT - ratio of total non-essential amino acids in adult to total amino acids, BI / BD - the ratio of total essential amino acids in adults to total non-essential amino acids, the same below 


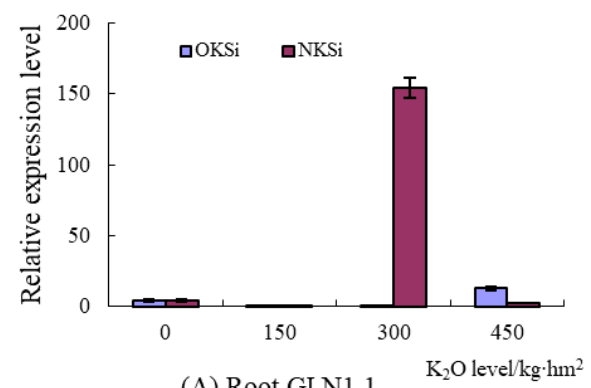

(A) Root GLN1.1

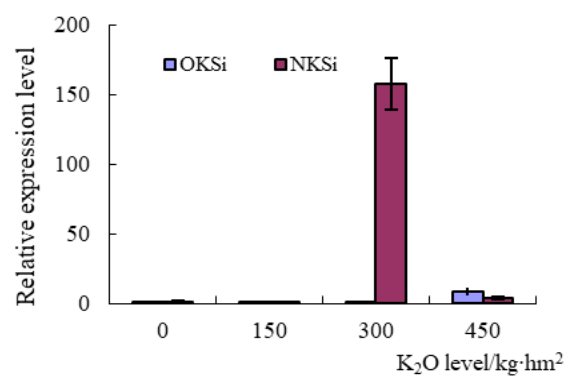

(C) Root GLN1.3

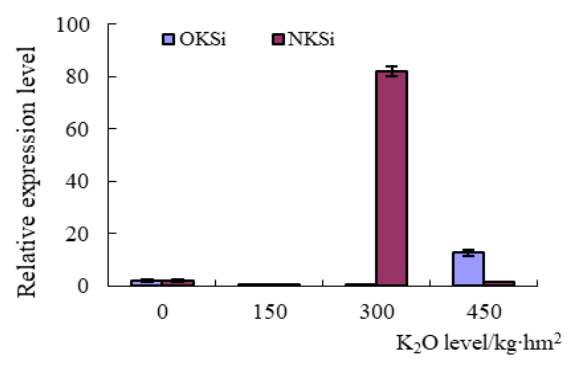

(B) Root GLN1.2

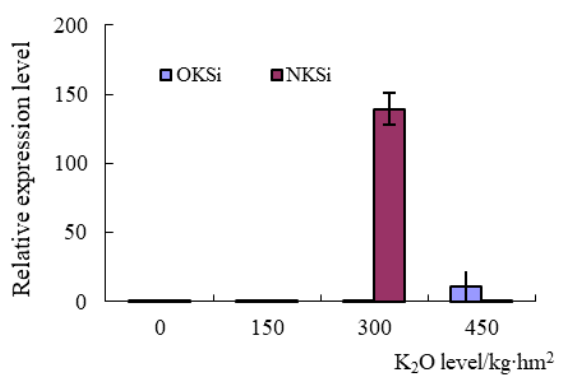

(D) Root GLN1.4

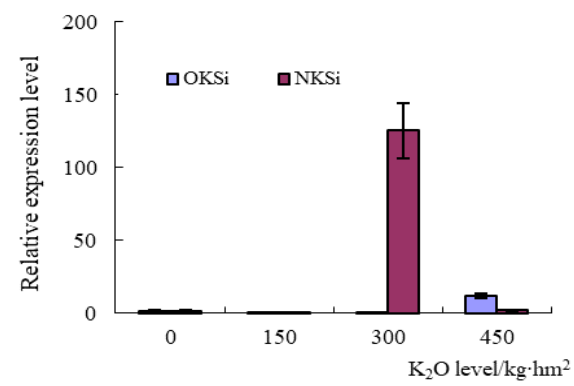

(E) Root GLN2

Figure 4. Relative expression of GLN family genes in Chinese cabbage root under different potassium silicate treatments

As can be seen from Figure 5, GLN family gene in Chinese cabbage stem (BrS) shows the following trend under potassium silicate treatment: under ordinary potassium silicate (OKSi) treatment, GLN1.1, GLN1.2 and GLN1.3 expressions are gradually downregulated with the increasing application rate, while GLN1.4 expression first decreases and then increases. The relative expression is the highest under OKSi-300 treatment, while that under OKSi-300 and OKSi-450 treatments is up by 1.16 and 0.088 times compared with the control (CK), respectively. GLN2 shows up-regulated expression, which is up by $1.79,10.1$ and 4.03 times compared with $\mathrm{CK}$, respectively, under application rates of 150,300 and $450 \mathrm{~kg} \cdot \mathrm{hm}^{-2}$. Under nano potassium silicate (NKSi) treatment, relative expressions of GLN1.1, GLN1.2, GLN1.3, GLN1.4 and GLN2 are upregulated under each treatment, showing higher increase range than that of $\mathrm{OKSi}$. The relative expression of GLN1.1 is the highest under NKSi-450 treatment, which is 0.603 times higher than CK. GLN1.2, GLN1.3, GLN1.4 and GLN2 have the highest relative expression under NKSi-300 treatment, which is up by 7.85, 3.46, 45.6 and 50.7 times, respectively compared with $\mathrm{CK}$, showing the order of expression of GLN1.4 $(1.30)<$ GLN1.2(3.38)<GLN1.3(4.46)< GLN2 (4.87). The above analysis indicates that NKSi treatment can significantly up-regulate GLN family gene expression in Chinese cabbage stem, with superior up-regulation effect than OKSi. 


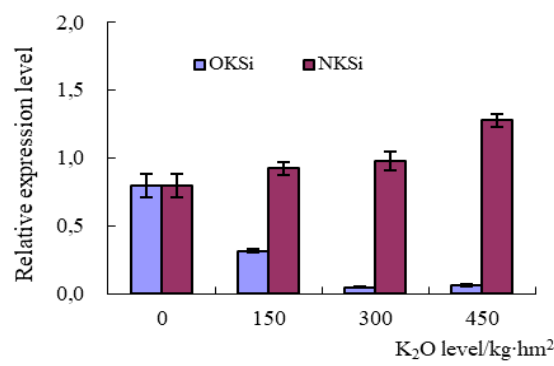

(A) Stem GLN1.1

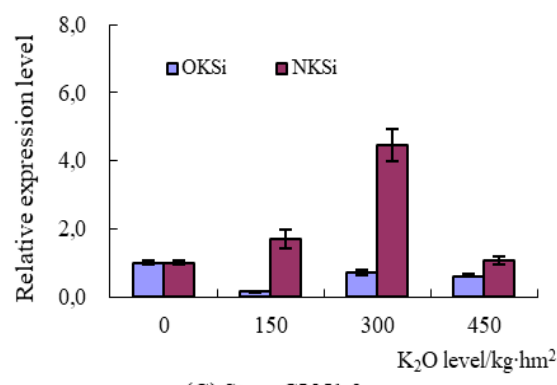

(C) Stem GLN1.3

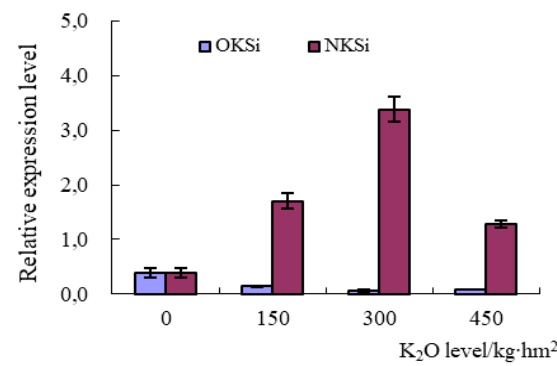

(B) Stem GLN1.2

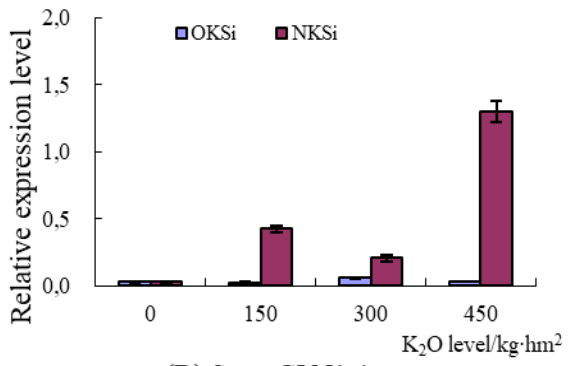

(D) Stem GLN1.4

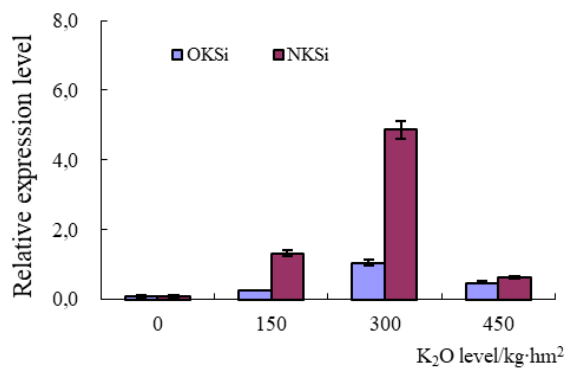

(E) Stem GLN2

Figure 5. Relative expression of GLN family genes in Chinese cabbage stem under different potassium silicatetreatments

As can be seen from Figure 6, GLN family gene shows the following trend in Chinese cabbage leaves $(\mathrm{BrL})$ under potassium silicate treatment: relative expression of GLN1.1, GLN1.2, GLN1.4 and GLN2 is gradually up-regulated with increasing application rate under ordinary potassium silicate $(\mathrm{OKSi})$ treatment, reaching the highest under OKSi-450 treatment, which is $2.19,6.29,1.63$ and 58.7 times higher than that of the control (CK), and the following order of expression of GLN1.4 (1.25)<GLN1.1 (2.07)<GLN1.2 $(6.12)<$ GLN2 (6.48) is shown. GLN1.3 expression is first down-regulated and then up-regulated, with the highest relative expression in OKSi-300 treatment, followed by OKSi-450, which is up by 9.59 and 8.96 times, respectively, compared with CK. Under nano potassium silicate (NKSi) treatment, expression of GLN1.1, GLN1.2 and GLN1.3 is first up-regulated and then down-regulated, showing the highest relative expression under NKSi-150 treatment, which is up by $0.984,0.235$ and 0.617 times, respectively, compared with CK. Only NKSi-150 brings higher expression level than OKSi-150, while other relative gene expression levels are higher in OKSi treatment under the same application rate. The relative expression of GLN1.4 is down-regulated while that of GLN2 is up-regulated, both of which are lower than that of OKSi treatment under the same application rate. The above analysis suggests that OKSi treatment can significantly up-regulate GLN family gene expression in Chinese cabbage leaves, showing superior up-regulation effect than NKSi. 


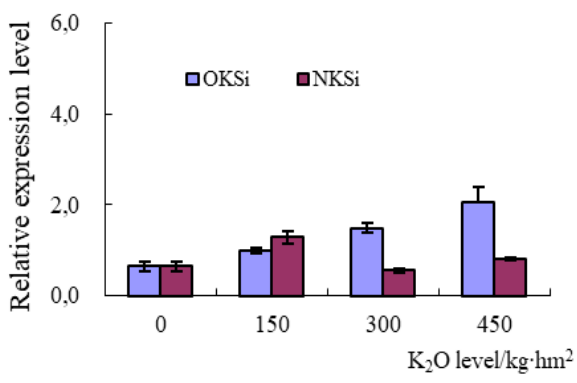

(A) Leaf GLN1.1

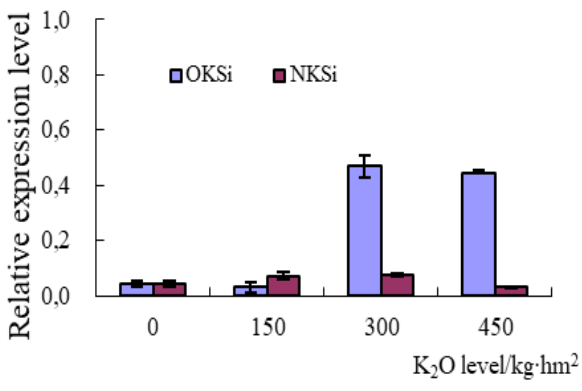

(C) Leaf GLN1.3

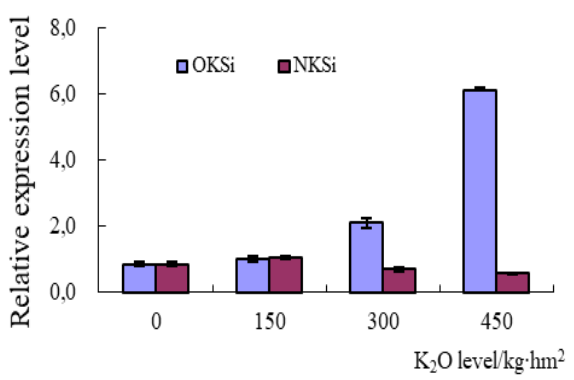

(B) Leaf GLN1.2

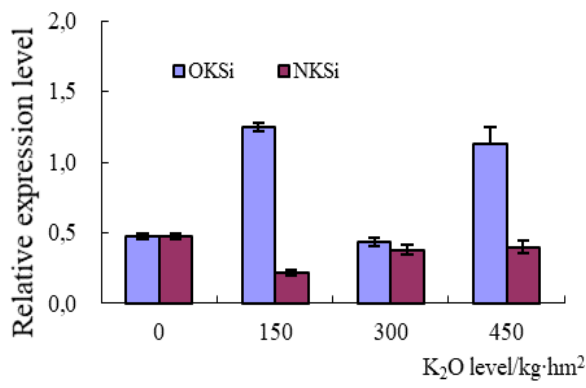

(D) Leaf GLN1.4

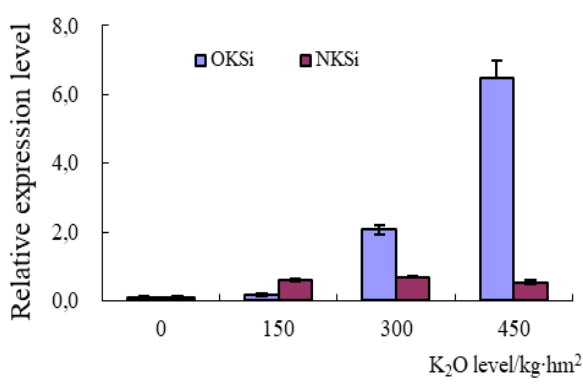

(E) Leaf GLN2

Figure 6. Relative expression of GLN family genes in Chinese cabbage leave under different potassium silicate treatments

\section{Correlation analysis between GLN family gene expression and amino acid component}

As can be seen from Table 9, BrRGLN1.1 ( $\mathrm{r}=0.779 *)$, BrRGLN1.2 ( $\mathrm{r}=0.794$ ), BrRGLN1.3 ( $\left.\mathrm{r}=0.784^{*}\right)$, BrRGLN1.4 $\left(\mathrm{r}=0.783^{*}\right)$ and BrRGLN2 $\left(\mathrm{r}=0.786^{*}\right)$ are significantly positively correlated with histidine (His), BrRGLN1.3 ( $\left.\mathrm{r}=0.759^{*}\right)$ is significantly positively correlated with glycine (Gly); BrSGLN1.1 ( $\mathrm{r}=-0.922 * *,-0.900 * *$ and $\left.-0.898^{* *}\right)$ is significantly negatively correlated with methionine (Met), tyrosine (Tyr) and cysteine (Cys), BrSGLN1.2 ( $\mathrm{r}=0.866^{*}$ and 0.820*), BrSGLN1.3 ( $\mathrm{r}=0.758^{*}$ and $\left.\left.0.775^{*}\right), \mathrm{BrSGLN} 1.4\left(\mathrm{r}=0.808^{*} \text { and } 0.811\right)^{*}\right)$ and BrSGLN2 $\left(\mathrm{r}=0.762^{*}\right.$ and $\left.0.781^{*}\right)$ are significantly positively correlated with lysine (Lys) and His, and BrSGLN1.2 $\left(r=-0.762^{*}\right.$ and $\left.-0.799^{*}\right)$ is significantly negatively correlated with Met and Cys, BrSGLN1.2 $\left(\mathrm{r}=0.780^{*}\right)$ and BrSGLN1.4 $\left(\mathrm{r}=0.763^{*}\right)$ are significantly positively correlated with Gly; BrLGLN1.2 $\left(\mathrm{r}=0.822^{*}\right.$ and $\left.0.757 *\right)$ and BrLGLN1.4 $\left(\mathrm{r}=0.834^{*}\right.$ and $\left.0.782 *\right)$ are significantly positively correlated with Met and Tyr, BrLGLN1.4 (r=0.757*) is significantly positively correlated with Cys, while other amino acids show no significant or highly significant correlation with GLN family gene. 
Table 9. Correlation coefficient ( $r$ ) between amino acid component (AAs) and GLN family genes in Chinese cabbage under different potassium silicate treatments

\begin{tabular}{|c|c|c|c|c|c|c|c|c|c|c|c|c|}
\hline \multirow{2}{*}{ Amino acids } & \multicolumn{12}{|c|}{ GLN family genes } \\
\hline & BrRGLN1.1 & BrRGLN1.2 & BrRGLN1.3 & BrRGLN1.4 & BrRGLN2 & BrSGLN1.1 & BrSGLN1.2 & BrSGLN1.3 & BrSGLN1.4 & BrSGLN2 & BrLGLN1.2 & BrLGLN1.4 \\
\hline Lys & 0.667 & 0.667 & 0.683 & 0.676 & 0.673 & 0.589 & $0.866^{*}$ & $0.758^{*}$ & $0.808^{*}$ & $0.762 *$ & -0.207 & -0.347 \\
\hline Met & -0.359 & -0.309 & -0.384 & -0.357 & -0.348 & $-0.922 * *$ & $-0.762 *$ & -0.597 & -0.595 & -0.473 & $0.822^{*}$ & $0.834 *$ \\
\hline Ile & 0.470 & 0.502 & 0.461 & 0.473 & 0.480 & -0.414 & 0.005 & 0.147 & 0.196 & 0.315 & 0.382 & 0.473 \\
\hline Leu & 0.708 & 0.743 & 0.701 & 0.714 & 0.720 & -0.236 & 0.335 & 0.471 & 0.510 & 0.617 & 0.379 & 0.405 \\
\hline Phe & 0.714 & 0.749 & 0.697 & 0.710 & 0.720 & -0.136 & 0.304 & 0.454 & 0.463 & 0.518 & 0.351 & 0.257 \\
\hline Val & 0.678 & 0.699 & 0.687 & 0.691 & 0.691 & 0.106 & 0.562 & 0.545 & 0.635 & 0.678 & 0.101 & 0.052 \\
\hline Thr & 0.327 & 0.348 & 0.336 & 0.331 & 0.337 & 0.205 & 0.245 & 0.177 & 0.241 & 0.255 & 0.091 & 0.096 \\
\hline His & $0.779 *$ & $0.794 *$ & $0.784 *$ & $0.783^{*}$ & $0.786^{*}$ & 0.480 & $0.820^{*}$ & $0.775^{*}$ & $0.811^{*}$ & $0.781^{*}$ & -0.017 & -0.199 \\
\hline Arg & 0.673 & 0.673 & 0.685 & 0.673 & 0.675 & 0.635 & 0.748 & 0.659 & 0.695 & 0.642 & -0.242 & -0.340 \\
\hline Glu & -0.450 & -0.430 & -0.465 & -0.457 & -0.449 & -0.639 & $-0.827 *$ & -0.716 & -0.721 & -0.636 & 0.389 & 0.559 \\
\hline Ser & 0.008 & 0.019 & 0.003 & 0.003 & 0.009 & -0.397 & -0.415 & -0.309 & -0.285 & -0.196 & 0.151 & 0.371 \\
\hline Gly & 0.749 & 0.754 & $0.759 *$ & 0.752 & 0.754 & 0.531 & $0.780^{*}$ & 0.714 & $0.763^{*}$ & 0.731 & -0.168 & -0.273 \\
\hline Ala & 0.481 & 0.495 & 0.491 & 0.483 & 0.487 & 0.581 & 0.598 & 0.459 & 0.515 & 0.441 & -0.085 & -0.288 \\
\hline Tyr & -0.224 & -0.177 & -0.250 & -0.223 & -0.215 & $-0.900 * *$ & -0.689 & -0.501 & -0.498 & -0.374 & $0.757 *$ & $0.782 *$ \\
\hline Pro & -0.290 & -0.305 & -0.280 & -0.278 & -0.287 & -0.665 & -0.537 & -0.471 & -0.397 & -0.268 & -0.032 & 0.309 \\
\hline Asp & 0.536 & 0.546 & 0.544 & 0.536 & 0.541 & 0.254 & 0.390 & 0.380 & 0.420 & 0.451 & -0.038 & 0.051 \\
\hline Cys & -0.388 & -0.351 & -0.403 & -0.383 & -0.378 & $-0.898 * *$ & $-0.799 *$ & -0.667 & -0.634 & -0.502 & 0.633 & $0.757 *$ \\
\hline BI & 0.697 & 0.730 & 0.696 & 0.705 & 0.710 & -0.013 & 0.468 & 0.513 & 0.575 & 0.636 & 0.289 & 0.225 \\
\hline $\mathrm{BD}$ & -0.044 & -0.024 & -0.048 & -0.043 & -0.038 & -0.493 & -0.452 & -0.368 & -0.318 & -0.213 & 0.279 & 0.474 \\
\hline BT & 0.282 & 0.312 & 0.279 & 0.287 & 0.293 & -0.358 & -0.112 & -0.032 & 0.032 & 0.136 & 0.337 & 0.451 \\
\hline
\end{tabular}




\section{Discussion}

Amino acids are important nutrients for vegetables. The content of amino acid components directly affects its nutritional value, which is closely related to human taste (Ou et al., 2007). Potassium is an activator of various enzymes in plant cells. Potassium deficiency affects nitrogen metabolism, which in turn affects the composition of free amino acids and total amino acids (Eppendorfer et al., 1996). In this study, the essential amino acids (BI), total non-essential amino acids (BD) and total amino acids (BT) of Chinese cabbage increased with the increasing application rate of ordinary potassium silicate $(\mathrm{OKSi})$ and nano potassium silicate $(\mathrm{NKSi})$. It indicates that proper application of potassium fertilizer contributes to the synthesis of amino acids in Chinese cabbage, which is similar to the research results of Tang et al. (2013). The mixture of salt compound of aspartic acid and glutamic acid and free acidic amino acids can lower the umami threshold with obvious synergistic effect and freshening effect, which is therefore known as umami amino acid, a major umami flavoring substance (Lioe et al., 2005). In this experiment, with the increasing application rate of ordinary potassium silicate (OKSi) and nano potassium silicate (NKSi), the concentration of umami amino acids showed an upward trend, suggesting that moderate application of potassium fertilizer in field experiment could improve content of umami amino acids in Chinese cabbage, which is consistent with the findings of Lin et al. (2003). On the whole, application of ordinary potassium silicate $(\mathrm{OKSi})$ and nano nanosilicate $(\mathrm{NKSi})$ can increase the content of sweet amino acids (glycine, alanine, threonine, and serine) in Chinese cabbage to some extent. Under NKSi-450treatment, glycine, alanine, threonine and serine were increased by $32.4 \%$, $33.4 \%, 42.3 \%$ and $0.8 \%$, respectively, compared with CK. The above results indicate that: under the experimental conditions of this study, application of potassium fertilizer can increase the total amount of amino acids in Chinese cabbage, greatly improve umami amino acids and sweet amino acids, thereby improving the nutritional value and flavor quality of Chinese cabbage. Nanotechnology has been applied to fertilizer modification and development of new fertilizers (Conesa et al., 2010; Adhikari et al., 2015). Thanks to the unique properties of nanomaterials, nanofertilizers have achieved good yield increase benefits in various crop applications. In the present study, NKSi-BI/BD was higher than OKSi-BI/BD under the same application rate, with the highest under NKSi-300 treatment. Principal component analysis method was used for dimensionality reduction and comprehensive evaluation of amino acids (AAs). The results showed that AAs comprehensive score was the highest under NKSi-300 treatment. The above analysis indicates that nano-potassium fertilizer has better benefits in improving amino acid components of Chinese cabbage and perfecting its flavor quality compared with traditional potassium fertilizer. It may be related to the fact that nano-fertilizer particles have better physical properties, which can enhance permeability of cell wall and cell membranes of plants, so that it is more easily absorbed by plant cells (Adhikari et al., 2015). This is similar to the finding by Lee et al. that mung bean and wheat have significant absorption effects on nano-copper (Lee et al., 2010).

Glutamine synthetase (GS) constitutes one of the key enzymes in the nitrogen metabolism pathway of higher plants (Feng et al., 2015). The ammonium directly absorbed by plants from the outside world and the ammonium obtained from nitrate reduction are further assimilated into amino acids via glutamine synthetase (GS)/ glutamate synthase (GOGAT) circulation (Lea and Miflin, 1974). Schuller et al. (1986) 
found that: potassium application could increase nitrogen metabolism-related key enzyme activities in soybean and Sesbania rostrata. The synthesis of biomacromolecules such as enzymes is regulated by gene expression. The activity of glutamine synthetase (GS) is subject to influence of the expression level of GLN family genes. It is one of the focuses of this study to determine the effect of treatment with different concentrations of $\mathrm{OKSi}$ and NKSi on GLN family genes of Chinese cabbage under field experimental conditions. In this study, application of ordinary potassium silicate (OKSi) and nano potassium silicate (NKSi) have a certain up-regulation effect on GLN family gene expression in the roots, stems and leaves of Chinese cabbage. NKSi and OKSi treatments have different effects on expression of GLN family genes in different parts of Chinese cabbage. NKSi can better up-regulate GLN family gene expression in roots and stems of Chinese cabbage than OKSi, but the latter has better up-regulation effect on leaves than NKSi. This may be because potassium promotes the uptake of NO3- in plant roots, thus accelerating the process of plant nitrogen metabolism, and promoting the synthesis of amino acids (Edward, 1981). The completion of nitrogen metabolism is inseparable from catalytic action of related key enzymes (GS, GOGAT, etc.). That is to say, potassium may upregulate GLN family gene expression by a certain mechanism to increase the content of nitrogen metabolism-related enzymes in roots and stems, thereby achieving efficient conversion of nitrogen. Compared with ordinary potassium fertilizer, Chinese cabbage has higher absorption rate of nano potassium fertilizer. NKSi treatment may increase absorption of potassium ions in roots and stems of Chinese cabbage, thus showing superior up-regulation effect on GLN family gene than OKSi. The main organ for plant uptake and transport of nitrogen is root system (Fan et al., 2010). NKSi can better upregulate GLN family genes in roots and stems of Chinese cabbage than OKSi, which means that NKSi has superior effect on root and stem vegetables than on leaf vegetables.

\section{Conclusion}

The ratio of essential amino acids to total amino acids (BI/BT) in Chinese cabbage increased with the increasing application rate of OKSi and NKSi, reaching the highest under NKSi-300 treatment. NKSi-BI/BD was higher than OKSi-BI/BD at the same application rate, with the highest under NKSi-300 treatment. NKSi treatment can significantly up-regulate GLN family genes in roots and stems of Chinese cabbage, showing superior up-regulation effect than $\mathrm{OKSi}$, while OKSi treatment can significantly up-regulate GLN family genes in Chinese cabbage leaves, showing superior upregulation effect than NKSi. There exists a significant or extremely significant correlation between GLN family gene expression in Chinese cabbage and the contents of histidine, glycine, methionine, tyrosine and cysteine in the stems of Chinese cabbage. Nano potassium silicate (NKSi) could increase the content of amino acid components in Chinese cabbage, showing better effect than ordinary potassium silicate $(\mathrm{OKSi})$ at the same application rate, and NKSi-300 was the most appropriate. NKSi treatment significantly up-regulated the expression of GLN family genes in roots and stems of Chinese cabbage, showing superior up-regulation effect than OKSi.

Nanotechnology is a new material and technology. With the development of material science, nano-fertilizer and other nano-products are being gradually applied to agricultural production. Compared to the traditional fertilization techniques, nanofertilizers improve plant production and nutrient use efficiency by consuming a small amount of resources (Kashyap et al., 2015). Furthermore, nanoparticles (NPs) can 
increase crop yield and nutritional value by promoting plant metabolism (Ghormade et al., 2011) due to their unique physicochemical properties. In recent research, the effects of nano-potassium fertilizer on amino acid composition and gene expression of GLN family in Chinese cabbage were discussed in detail. However, the research of nanofertilizer on the physiological mechanism of crop, especially the micro-mechanism, needs to be strengthened. On the other hand, it has been reported that some nano-fertilizers show some toxicity to crops (Gui et al., 2015; Rico et al., 2017), so it is important to carry out toxicological research on nano-fertilizers. The emergence of nano-fertilizer plays an important role in promoting and innovating agricultural production mode, but there are still a lot of research on nano-fertilizer.

Acknowledgments. This work was supported by Fund of China Agriculture Research System (CARS-23), and the National Key Research and Development Program of China (2018YFD0201200), and the National College students Innovation and Entrepreneurship training Program (201910635054).

\section{REFERENCES}

[1] Adhikari, T., Kundu, S., Biswas, A. K., Tarafdar, J. C., Subba Rao, A. (2015): Characterization of zinc oxide nano particles and their effect on growth of maize (Zea mays L.) plant. - Journal of Plant Nutrition 38(10): 1505-1515.

[2] Conesa, H. M., Wieser, M., Gasser, M., Hockmann, K., Evangelou, M. W. H., Studer, B., Schulin, R. (2010): Effects of three amendments on extractability and fractionation of $\mathrm{Pb}$, $\mathrm{Cu}, \mathrm{Ni}$ and $\mathrm{Sb}$ in two shooting range soils. - Journal of Hazardous Materials 181(1-3): 845850 .

[3] Davarpanah, S., Tehranifar, A., Davarynejad, G., Aran, M., Abadía, J., Khorassani, R. (2017): Effects of foliar nano-nitrogen and urea fertilizers on the physical and chemical properties of pomegranate (Punica granatum cv. Ardestani) fruits. - HortScience 52(2): 288-294.

[4] Edward, E. (1981): Potash Fertilizer and Increased Tolerance to Stress. - Agriviews 1, No. I. Canada. Foth, R. D.

[5] Eppendorfer, W. H., Bille, S. W. (1996): Free and total amino acid composition of edible parts of beans, kale, spinach, cauliflower and potatoes as influenced by nitrogen fertilization and phosphorus and potassium deficiency. - Journal of the Science of Food and Agriculture 71(4): 449-458.

[6] Fan, J. B., Zhang, Y. L., Turner, D., Duan, Y. H., Wang, D. S., Shen, Q. R. (2010): Root physiological and morphological characteristics of two rice cultivars with different nitrogen-use efficiency. - Pedosphere 20(4): 446-455.

[7] Feng, W. J., Xing, G. F., Niu, X. L., Dou, C., Han, Y. H. (2015): Progress and application prospects of glutamine synthetase in plants. - Chin J Biotech 31: 1301-1312.

[8] Gascuel, Q., Diretto, G., Monforte, A. J., Fortes, A. M., Granell, A. (2017): Use of natural diversity and biotechnology to increase the quality and nutritional content of tomato and grape. - Frontiers in Plant Science 8: 1-24.

[9] Ghormade, V., Deshpande, M. V., Paknikar, K. M. (2011): Perspectives for nanobiotechnology enabled protection and nutrition of plants. - Biotechnology Advances 29(6): 792-803.

[10] Gui, X., Zhang, Z. Y., Liu, S. T., Ma, Y. H., Zhang, P., He, X., Li, Y. Y., Zhang, J., Li, H. F., Rui, Y. K., Liu, L. M., Cao, W. D. (2015): Fate and phytotoxicity of $\mathrm{CeO} 2$ nanoparticles on lettuce cultured in the potting soil environment. - Plos One 10(8): e0134261. 
[11] Huang, S., Wang, L., Liu, L., Hou, Y., Li, L. (2014): Nanotechnology in agriculture, livestock, and aquaculture in China. A review. - Agronomy for Sustainable Development 35(2): 369-400.

[12] Kashyap, P. L., Xiang, X., Heiden, P. (2015): Chitosan nanoparticle based delivery systems for sustainable agriculture. - International Journal of Biological Macromolecules 77: 3651.

[13] Lea, P. J., Miflin, B. J. (1974): Alternative route for nitrogen assimilation in higher plants. - Nature 251(5476): 614-616.

[14] Lee, C. W., Mahendra, S., Zodrow, K., Li, D., Tsai, Y. C., Braam, J., Alvarez, P. J. J. (2010): Developmental phytotoxicity of metal oxide nanoparticles to Arabidopsis thaliana. - Environmental Toxicology and Chemistry 29(3): 669-675.

[15] Li, T., Sun, F. Y., Gong, P., Wang, A., Yuan, L. X., Yin, X. B. (2017): Effects of Nanoselenium fertilization on selenium concentration of wheat grains and quality-related traits. - Journal of Plant Nutrition and Fertilizer 23(02): 427-433.

[16] Li, Y. H., Qin, Y. L., Xu, W. H., Chai, Y. R., Chi, S. L., Li, T., Zhang, C. L., Yang, M., He, Z. M., Feng, D. Y. (2019): Differences of Cd uptake and expression of MT and NRAMP2 genes in two varieties of ryegrasses. - Environmental Science and Pollution Research 26: 13738-13745.

[17] Lim, W., Miller, R., Park, J., Park, S. (2014): Consumer sensory analysis of high flavonoid transgenic tomatoes. - Journal of Food Science 79(6): S1212-S1217.

[18] Lin, D., Huang, D. F. (2003): Effects of potassium levels on photosynthesis and fruit quality of muskmelon in medium culture. - Acta Horticulturae Sinica 30: 221-223.

[19] Lioe, H. N., Apriyantono, A., Takara, K., Wada, K., Yasuda, M. (2005): Umami taste enhancement of MSG/NaCl mixtures by subthreshold $\mathrm{L}-\alpha$-Aromatic amino acids. - Journal of Food Science 70(7): s401-s405.

[20] Mattoo, A., Nath, P., Bouzayen, M. (2014): Fruit Ripening: Physiology, Signalling and Genomics. - CABI, Oxfordshire.

[21] Ou, X. Q., Ren, X. J., Zhou, Y. (2017): Analysis on the amino acid content and the composition in the vegetable sweet potato tips. - Journal of Chinese Institute of Food Science and Technology 7: 120-125.

[22] Rico, C. M., Johnson, M. G., Marcus, M. A., Andersen, C. P. (2017): Intergenerational responses of wheat (Triticum aestivum L.) to cerium oxide nanoparticles exposure. Environmental Science: Nano 4(3): 700-711.

[23] Schuller, K. A., Day, D. A., Gibson, A. H., Gresshoff, P. M. (1986): Enzymes of ammonia assimilation and ureide biosynthesis in soybean nodules: effect of nitrate. - Plant Physiology 80: 646-50.

[24] Wang, X. J., Chen, Y., Wang, F., Wang, Z. Y. (2015): Effects of potash fertilizer on cabbage's quality in cadmium polluted soils. - Journal of Agricultural Resources and Environment 32(1): 40-47.

[25] Yuan, T., Wang, Z. Y., Gu, S. K., Wang, F., Yang, D., Cheng, Y. (2017): Effect of combined application of low-level potassium fertilizer with nano- $\mathrm{Mg}(\mathrm{OH}) 2$ on Chinese cabbage quality. - Journal of Plant Nutrition and Fertilizer 23: 254. 